\title{
Development of a Field-Portable Small-Size Impedance Analyzer for Structural Health Monitoring using the Electromechanical Impedance Technique
}

\author{
Victor Giurgiutiu, $\mathrm{PhD}$, \\ Mechanical Engineering Department, University of South Carolina \\ Columbia, SC 29208, victorg@sc.edu \\ Buli Xu, \\ Mechanical Engineering Department, University of South Carolina \\ Columbia, SC 29208, xub@engr.sc.edu
}

\begin{abstract}
Electromechanical (E/M) impedance method is emerging as an effective and powerful technique for structural health monitoring. The $\mathrm{E} / \mathrm{M}$ impedance method utilizes as its main apparatus an impedance analyzer that reads the in-situ E/M impedance of piezoelectric wafer active sensors (PWAS) attached to the monitored structure. Laboratory-type impedance analyzers (e.g. HP4194) are bulky, heavy, and expensive. They cannot be easily carried into the field for onsite structural health monitoring. To address this issue, means of to reduce the size of the impedance analyzer making the impedance analyzer more compact and field-portable are explored. In this paper, we present a systematic approach to the development of a field-portable small-size impedance analyzer for structural health monitoring using the electromechanical impedance technique. Our approach consists of several developmental stages. First, we perform a simulation of the E/M Impedance technique and develop the software tools for analyzing the signal in a fast and efficient way while maintaining the desired accuracy. The objective of this signal processing part is to obtain the complex impedance, $\left.Z_{\mathrm{R}}+\mathrm{i} \mathrm{Z}_{\mathrm{I}}\right)=|\mathrm{Z}| \angle \arg Z$, at a number of frequencies in a predetermined range. Several signal processing methods were explored such as: (a) integration method; (b) correlation method; (c) Discrete Fourier transform (DFT) method. Second, we discuss the hardware issues associated with the implementation of this approach. The hardware system architecture consists of several blocks: (a) reference signal generation; (b) voltage and current measurements; and (c) digital signal acquisition and processing. Practical results obtained during proof-of-concept experiments are presented and comparatively examined.
\end{abstract}

Keywords: Electro-Mechanical impedance method, PWAS, integration, correlation, DFT, simulation

\section{INTRODUCTION}

\subsection{Background}

Structural health monitoring (SHM) is a method of determining the health of a structure from the readings of an array of permanently-attached sensors that are embedded into the structure and monitored over time. SHM can be performed in basically two ways, passive and active. Passive SHM consists of monitoring a number of parameters (loading stress, environment action, performance indicators, acoustic emission from cracks, etc.) and inferring the state of structural health from a structural model. In contrast, active SHM performs proactive interrogation of the structure, detects damage, and determines the state of structural health from the evaluation of damage extend and intensity. Both approaches aim at performing a diagnosis of the structural safety and health, to be followed by a prognosis of the remaining life. Passive SHM uses passive sensors which only "listen" but do not interact with the structure. Therefore, 
they do not provide direct measurement of the damage presence and intensity. Active SHM uses active sensors that interact with the structure and thus determine the presence or absence of damage. The methods used for active SHM resemble the methods of nondestructive evaluation (NDE), e.g., ultrasonics, eddy currents, etc., only that they are used with embedded sensors. Hence, the active SHM could be seen as "embedded NDE". One widely used active SHM method employs piezoelectric wafer active sensors (PWAS), which send and receive Lamb waves and determine the presence of cracks, delaminations, disbonds, and corrosion. Due to its similarities to NDE ultrasonics, this approach is also known as "embedded ultrasonics".
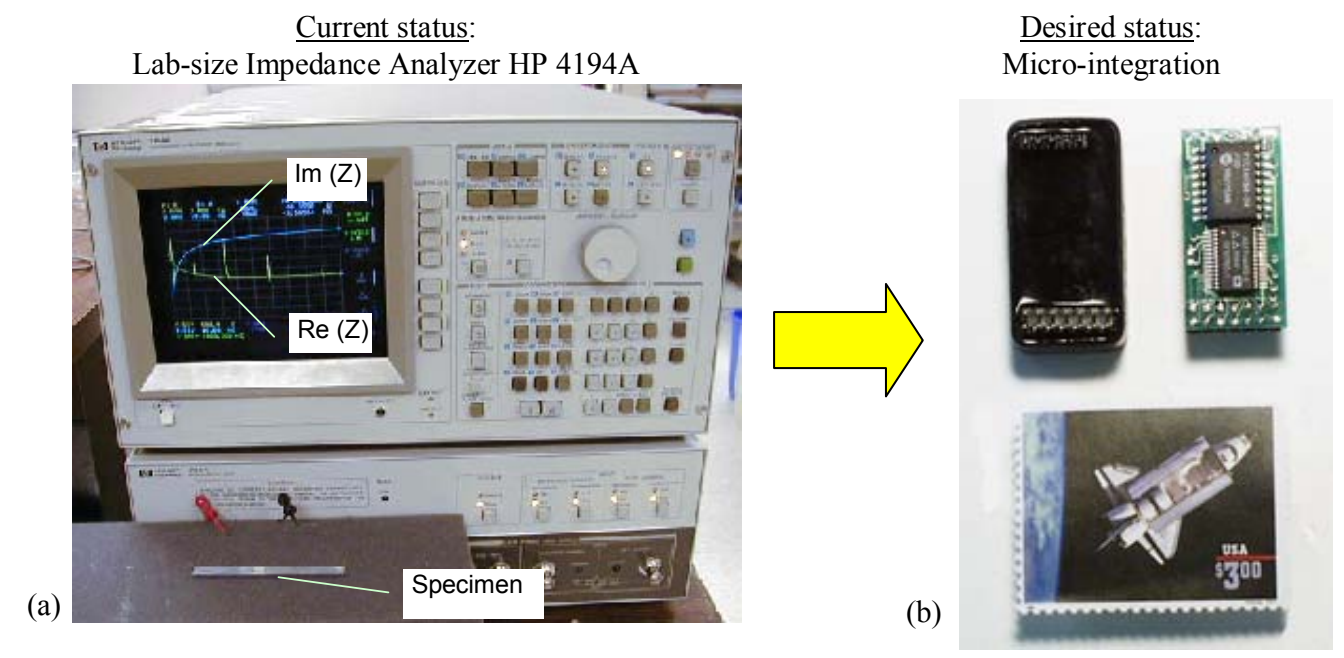

Figure 1 Miniaturization and integration of PWAS SHM equipment: (a) existing lab size impedance analyzer (bulky and relatively expensive); (b) miniaturized integrated electronics DAQ and tele-transmitter IC's (StrainLink, www.microstrain.com) that represent the miniaturization level targeted in this paper

The electromechanical (E/M) impedance method is a embedded ultrasonic method that is emerging as an effective and powerful technique for SHM. Through PWAS permanently attached to the structure, the E/M impedance method is able to measure directly the high-frequency local impedance spectrum of the structure. Because the high-frequency local impedance spectrum is much more sensitive to incipient damage than the low-frequency global impedance, the E/M impedance method is better suited for applications in structural health monitoring then other more conventional methods. The E/M impedance method utilizes as its main apparatus an impedance analyzer that reads the in-situ E/M impedance of the PWAS attached to the monitored structure.

\subsection{Motivation}

Although PWAS are small, unobtrusive, and inexpensive, the laboratory measurement equipment used in the proof-ofconcept demonstration of this technology is bulky, heavy, and relatively expensive. Laboratory-type impedance analyzers (e.g. HP4194A) cost around $\$ 40,000$ and weigh around $40 \mathrm{~kg}$. They cannot be easily carried into the field for on-site structural health monitoring. Therefore, such equipment is improper for large-scale deployment of the E/M impedance technology for SHM applications. In contrast, the PWAS transducers cost only around $\$ 20$ each, are small, unobtrusive, lightweight, and low power. It is apparent that in order to reach the long-term SHM goals and achieve wide industrial dissemination, the electronic equipment must be miniaturized and its cost must be reduced to a level similar to that of the PWAS transducers.

The objective of this paper is to present an integrated electronics concept for PWAS E/M impedance SHM -- compact $\mathrm{E} / \mathrm{M}$ impedance analyzer ${ }^{[1]}$. The general aim is to go from the present-day bulky and expensive laboratory-size impedance analyzers to an integrated-circuit impedance analyzer that can be easily embedded into a structure for in-situ SHM (Figure 1). This objective is achieved by simplifying, miniaturizing, and integrating the functionality of existing laboratory-size electrical-impedance measuring equipment and by developing new implementation concepts utilizing 
state-of-the-art methods and technologies in on-chip data acquisition, processing, and teletransmission. We believe that this miniaturization is possible because:

1. The E/M impedance technique uses only a very small subset of all the functions and capabilities of laboratoryscale impedance analyzers such as the HP4194A instrument.

2. The design of existing laboratory-scale impedance analyzers is based on 1970's technology, while the design options considered in the present paper will utilize the latest developments in signal processing and microelectronics, many of them associated with the remarkable progress recently achieved in wireless communication.

The implementation of this miniaturized impedance analyzer in active SHM systems will result in the seamless integration of active sensing, electronics, analysis, and diagnostics, into a compact system in an unobtrusive way. In addition, by developing a lost-cost miniaturized impedance-measuring device, an on-line SHM system would be more compact and more easily used by the maintenance crews and SHM operators.

\section{STATE OF THE ART}

\subsection{Impedance measurement methods}

\subsubsection{What is impedance?}

Impedance is an important parameter used to characterize electronic circuits, components, and the materials used to make components. Impedance $(Z)$ is generally defined as the total opposition a device or circuit offers to the flow of an alternating current (AC) at given frequency, and is represented as a complex quantity. An impedance vector $Z$ consists of a real part (resistance, R) and an imaginary part (reactance, $X$ ). To find the impedance, we need to measure at least two values since impedance is a complex quantity ${ }^{[2]}$.

\subsubsection{Impedance analyzer}

There are many measurement methods to choose from when measuring impedance, such as, RC bridge method, resonant method, auto balancing bridge method, network analysis method, I-V method, etc. Each of these methods has advantages and disadvantages ${ }^{[2]}$. Auto balancing bridge method (Figure 2 a) and I-V method (Figure 2 b) are the methods that can measure impedance within a wide frequency range.

In the auto balancing bridge method, the current, flowing through the device under test (DUT) also flows through the resistor $R$. The potential at the $\mathrm{L}$ point is maintained at zero volts (thus called a "virtual ground"), because the current through $R$ balances with the current through the DUT through the use of an IV converter amplifier. The DUT impedance is calculated using voltage measurements at the high terminal $\mathrm{H}$ and across the resistance $R$. In real application, a more complicated circuit is used.

In the I-V method, an unknown impedance $Z_{x}$ can be calculated from the measured voltage and current. Current is calculated using the voltage measurement across an accurately known low value resistor, R. This method is comparatively simpler than the auto balancing bridge method.

(a)

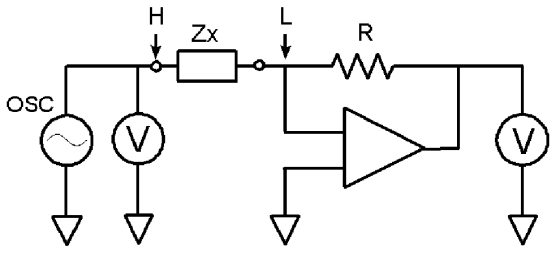

(b)

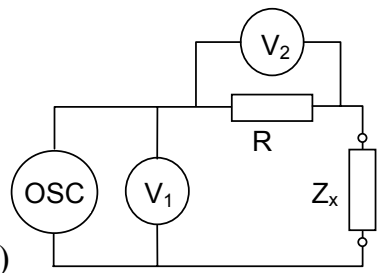

Figure 2 Impedance measurement methods ${ }^{[2]}$ : (a) I-V method; (b) auto balancing bridge method

There are several companies working on impedance analyzer development: Quatech ${ }^{[3]}$ (using discrete fourier transform method to measure large impedance values), Solatron ${ }^{[4]}$ (using sine correlation method to measure electrochemical impedance), Agilent ${ }^{[2]}$ (using sine correlation method to measure large impedance values), and Gamry ${ }^{[5]}$ (using sub- 
harmonic sampling method to measure electrochemical impedance), etc. However, all these developments refer to laboratory-scale impedance analyzers, which are bulky and expensive.

\subsection{Results from other investigators}

Several investigators have explored means of reducing the size of the impedance analyzer, to make it more compact, and even field-portable. Alternative ways of measuring the E/M impedance, which are different from those used by the impedance analyzer, have also been considered.

Pardo de Vera and Guemes ${ }^{[6]}$ employed the electromechanical (E/M) impedance method to detect damage in a GFRP composite specimen using a simplified impedance measuring technique. The simplified impedance-measuring technique consisted in the use of an inexpensive laboratory-made RC-bridge instead of the costly HP4194A impedance analyzer (Figure 3 a).

The disadvantages of using the RC-bridge are: (a) Additional instrumentation and processing needs to be used to separate the signal into its real (in phase) part and imaginary (quadrature) part. (b) Precise bridge balance needs to be initially attained in order to prevent the excitation signal from leaking into the output and masking the sensing signals.

(c) Need to be manually balanced. (d) Narrow frequency coverage with a single instrument ${ }^{[2]}$.

(a)

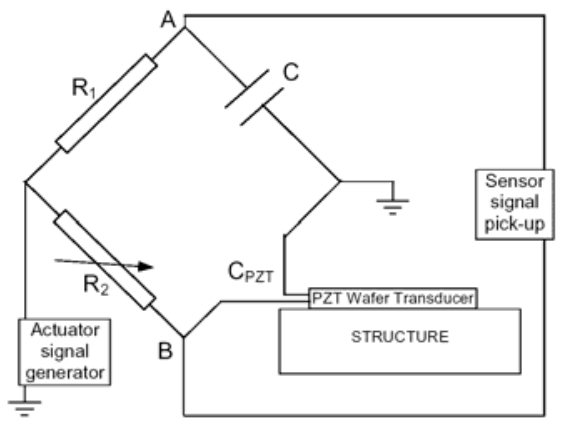

Figure 3

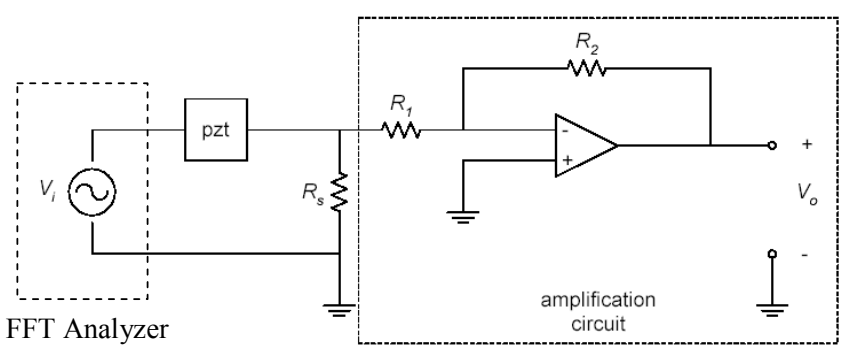

(b)

Previous results: (a) Inexpensive $\mathrm{RC}$ bridge ${ }^{[6]}$ for detecting the E/M impedance change of a PZT wa
affixed to the health monitored structure; (b) Impedance approximating circuit with amplification ${ }^{[7,8,9,10]}$

Peairs, Park and Inman ${ }^{[7,8,9,10]}$ suggested a method of generating impedance measurements utilizing an FFT analyzer and a small current-measuring circuit (Figure $3 \mathrm{~b}$ ). The approximated impedance $\left(Z_{\mathrm{PZT}}\right)$ is: $Z_{P Z T}=R_{s}\left(V_{i}-V_{0}\right) / V_{0}$.

The disadvantages of this method are: (a) Inaccuracy at high frequencies due to the performance limits of the amplifier. (b) A large laboratory-scale instrument-FFT analyzer is still needed. (c) Currently available FFT analyzer is only appropriate for the analysis of signals in the range of a few millihertz to about a hundred kilohertz ${ }^{[1]}$, which is insufficient for many SHM applications.

Darowicki, $K{ }^{\left[{ }^{[12]}\right.}$ presented the mathematical basis of continuous-frequency method to measure the impedance of an electrode. The method uses a non-stationary perturbation signal whose frequency varies linearly with time. The recording and analysis of both the perturbation and response signals allow the determination of continuous-frequency impedance spectra.

\section{THE CONCEPT AND THEORETICAL BASIS}

\subsection{The Concept}

The method presented in this paper is to take a systematic approach to the development of the field-portable compact small-size impedance analyzer for structural health monitoring using the E/M impedance technique. Our approach consists of several developmental stages:

- First, we simulated the E/M impedance technique and developed the software tools for analyzing the signal in a fast and efficient way while maintaining the desired accuracy. The objective of this part is to obtain the 
complex impedance $\left.\mathrm{Z}_{\mathrm{R}}+\mathrm{i} \mathrm{Z}_{\mathrm{I}}\right)=|\mathrm{Z}| \angle \arg Z$ at a number of frequencies in a predetermined range. Several signal processing methods are explore ${ }^{[13]}$ such as:
(1) Integration
(2) Correlation
(3) Discrete Fourier transform (DFT)

- Second, we analyzed the hardware issues associated with the implementation of this approach. The size of the overall system was considered, and implementations using either a laptop or a digital signal processor (DSP) are considered. The system architecture consisted of several blocks:
(1) Reference signal generation
(2) Voltage and current measurements
(3) Digital signal acquisition and processing

- Third, we performed a thorough calibration of our new methodology using an active component, the PWAS. In this calibration, we compared the results measured with our new method against the results measured with an existing laboratory-size impedance analyzer (e.g., HP4194A).

Details of our method of approach are given next. Practical results obtained during proof-of-concept experiments are also presented and comparatively examined.

\subsection{Theoretical Basis of the Compact E/M Impedance Analyzer}

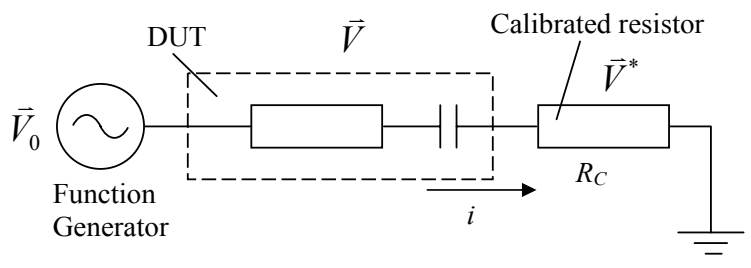

Figure 4 Schematic of the impedance measuring principle used in our work

Compact E/M impedance analyzer ${ }^{[1]}$ utilizes the I-V method for its simplicity of measuring circuit and wide frequency range. The circuit in Figure 4 used to measure the impedance of the device under test (DUT). This circuit is similar to a voltage divider. The function generator outputs sinusoidal excitation $\vec{V}_{0}$ within a predefined frequency range (from $f_{\text {start }}$ to $f_{\text {end }}$, say, $100 \mathrm{KHz}$ to $1 \mathrm{MHz}$ ). The current $i$ flowing through the DUT also flows through the known low-value calibrated resistor $R_{c}$ (say, $R_{c}=100 \Omega$ ). The current $i$ is calculated using the voltage $\vec{V}^{*}$ measured across the calibrated resistor $R_{c}\left(i=\vec{V}^{*} / R_{c}\right)$. Hence, the DUT impedance is given by

$$
Z=\frac{\vec{V}}{i}=\frac{\vec{V}}{\vec{V}^{*}} R_{c}
$$

Where, $Z$ is the impedance of DUT, $i$ is the current, $\vec{V}^{*}$ is the voltage across resistor $R_{c}$, and $\vec{V}$ is the voltage across the DUT. Note that the calculated impedance $Z$ may be change in both amplitude and phase with respect to the input. The impedance is a complex number. $\vec{V}$ and $\vec{V}^{*}$ are vectors and should be measured simultaneously. Impedance measurement depends on finding a method to measure vector signals.

\section{INTEGRATION APPROACH}

Suppose a vector voltage $x(t)=A \sin \left(\omega t+\varphi_{0}\right)$ is multiplied by sine signal and cosine signal respectively, and then 
integrated over a time duration $T$. If $T$ is the period of signal $x(t)$, the outputs will be the real part, Acos $\left(\varphi_{0}\right)$, and the imaginary part, $\operatorname{Asin}\left(\varphi_{0}\right)$, of this harmonic voltage $x(t)$.

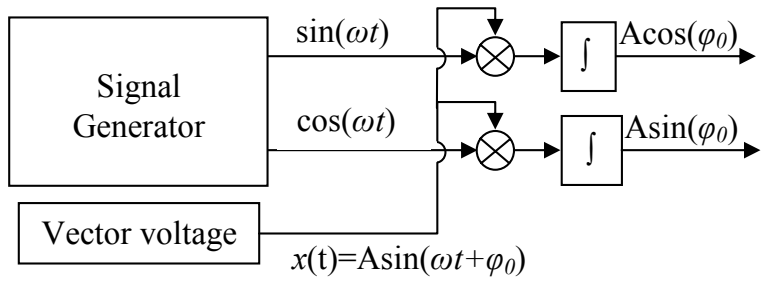

Figure 5 Schematic of the impedance measuring principle using the integration method

We can get this result from the calculation of the coefficient $c_{1}$ of complex Fourier series expansion of the signal $x(t)$, i.e.,

$$
\begin{array}{cc}
x(t)=\sum_{k=-\infty}^{\infty} c_{k} e^{j k \omega t} & -\infty<t<\infty \\
c_{k}=\frac{1}{T} \int_{0}^{T} x(t) e^{-j k \omega t} d t & k=0, \pm 1, \pm 2, \ldots
\end{array}
$$

Where, $T$ is the period of the signal $x(t), \omega$ is the angular frequency of $x(t)$, and $c_{k}$ are the coefficients of the Fourier series. Let $x(t)=A e^{j\left(\omega t+\varphi_{0}\right)}$. Then,

Equation (4) yields

$$
\begin{aligned}
c_{1} & =\frac{1}{T} \int_{0}^{T} x(t) e^{-j \omega t} d t \\
& =\frac{1}{T} \int_{0}^{T} A\left[\cos \left(\omega t+\varphi_{0}\right)+j \sin \left(\omega t+\varphi_{0}\right)\right] e^{-j \omega t} d t=A e^{j \varphi_{0}}
\end{aligned}
$$

i.e.,

$$
\frac{1}{T} \int_{0}^{T} j A \sin \left(\omega t+\varphi_{0}\right) e^{-j \omega t} d t=\frac{1}{2} A e^{j \varphi_{0}}
$$

$$
\begin{aligned}
& A \cos \varphi_{0}=\frac{2}{T} \int_{0}^{T} A \sin \left(\omega t+\varphi_{0}\right) \sin (\omega t) d t \\
& A \sin \varphi_{0}=\frac{2}{T} \int_{0}^{T} A \sin \left(\omega t+\varphi_{0}\right) \cos (\omega t) d t
\end{aligned}
$$

\section{CORRELATION APPROACH}

Consider two signals of the form:

$$
\begin{aligned}
& x(t)=A \sin \left(\omega t+\varphi_{0}\right)+N_{x}(t) \\
& y(t)=B \sin \left(\omega t+\varphi_{1}\right)+N_{y}(t)
\end{aligned}
$$

Where, $A$ is the amplitude of signal $x(t), B$ is the amplitude of signal $y(t), N_{x}(t)$ is the noise in signal $\mathrm{x}(t), N_{y}(t)$ is the noise in signal $y(t), \varphi_{1}$ is the initial phase of signal $x(t), \varphi_{2}$ is the initial phase of signal $y(t)$. The correlation of signals $x(t)$ and $y(t)$ is given by

$$
R_{X Y}(\tau)=\frac{1}{T} \int_{0}^{T} x(t) y(t-\tau) d t=\frac{1}{T} \int_{0}^{T}\left[A \sin \left(\omega t+\varphi_{0}\right)+N_{x}(t)\right] \cdot\left[B \sin \left(\omega(t-\tau)+\varphi_{1}\right)+N_{y}(t-\tau)\right] d t
$$

Where $\mathrm{T}$ is the time interval over which the correlation is required and $\tau$ is a time displacement or dealy

When $\tau=0$, 


$$
R_{X Y}(0)=\frac{1}{T} \int_{0}^{T}\left[A \sin \left(\omega t+\varphi_{0}\right)+N_{x}(t)\right] \cdot\left[B \sin \left(\omega t+\varphi_{1}\right)+N_{y}(t)\right] d t
$$

The signals, and noises $N_{x}(t)$ and $N_{y}(t)$, are not correlated. Hence,

$$
R_{X Y}(0)=\frac{A \cdot B}{2} \cos \left(\varphi_{1}-\varphi_{0}\right)=\frac{A \cdot B}{2} \cos \varphi
$$

Where the phase difference is $\varphi \doteq \varphi_{1}-\varphi_{0}$. Rearrange Equation(12) as

$$
\varphi=\arccos \frac{R_{X Y}(0)}{\sqrt{R_{X X}(0) \cdot R_{Y Y}(0)}}
$$

Equation (12) can be also used to find the amplitudes $A$ and $B$ using the autocorrelation function $R_{\mathrm{xx}}$ and $R_{\mathrm{YY}}$, i.e.,

$$
\begin{aligned}
& A=\sqrt{2 R_{X X}(0)} \\
& B=\sqrt{2 R_{Y Y}(0)}
\end{aligned}
$$

In the discrete-time domain, we get

$$
\begin{gathered}
R_{X Y}(0)=\frac{1}{N} \sum_{n=0}^{N-1} x(n) y(n) \\
R_{X X}(0)=\frac{1}{N} \sum_{n=0}^{N-1} x(n)^{2} \\
R_{Y Y}(0)=\frac{1}{N} \sum_{n=0}^{N-1} y(n)^{2}
\end{gathered}
$$

where, $N$ is the number of samples. Equations (13), (14) and (15) permit the calculation of the amplitude ratio and relative phase difference of the two vectors of Equation (1).

\section{DISCRETE FOURIER TRANSFORM APPROACH}

\subsection{Discrete Fourier Transform (DFT) Method}

Consider a harmonic signal:

$$
x_{1}(t)=A \sin (2 \pi f t)
$$

Where, $A$ is the signal amplitude and $f$ is the signal frequency. After sampling, the digitized signal of $x_{1}(t)$ is $x_{1}(n)$ given by:

$$
x_{1}(n)=A \sin \left(2 \pi f n T_{S}\right)=A \sin \left(2 \pi n f / f_{S}\right)=A \sin (2 \pi q n / N), \quad n=0,1,2, \ldots, N-1
$$

Where, $q=f N / f_{s}, N$ is the number of samples, $f_{S}$ is the sampling frequency, $T_{S}$ is the sampling time interval, $T_{S}=1 / f_{S}$. The Discrete Fourier Transform (DFT) of $x_{1}(n)$ is given by

$$
\begin{aligned}
X_{1}(K) & =\sum_{n=0}^{N-1} x(n) e^{-j(2 \pi / N) k n}=\sum_{n=0}^{N-1} A \sin (2 q n / N) e^{-j(2 \pi / N) k n} \\
& =A \frac{j}{2} \sum_{n=0}^{N-1}\left[e^{-j(2 \pi(q+k) n / N}-e^{-j(2 \pi(q-k) n / N}\right]
\end{aligned}
$$

Where, $k=0,1,2, \ldots, N-1$, and $j=\sqrt{-1}$. Note that:

$$
\begin{array}{ll}
k \neq q, & N-q ; \quad X_{1}(k)=0 \\
k=q, & N-q ; \quad X_{1}(k)=-\frac{A}{2} N j
\end{array}
$$

Now consider a harmonic signal with phase $\varphi$, i.e., 


$$
x_{2}(t)=A \sin (2 \pi f t+\varphi)
$$

Where, $\varphi$ is the initial phase of the signal. Performing the DFT of $x_{2}(t)$ gives

$$
X_{2}(k)=A \frac{j}{2} e^{j \varphi}\left(e^{-j 2 \pi(q+k) n / N}-e^{-j 2 \pi(q-k) n / N}\right), k=0,1,2, \ldots, \mathrm{N}-1
$$

Note that:

$$
\begin{array}{ccc}
k \neq q, & N-q ; & X_{2}(k)=0 \\
k=q, & N-q ; & X_{2}(k)=\frac{A}{2} N \sin \varphi-j \frac{A}{2} N \cos \varphi
\end{array}
$$

Equation (25) allows us to calculate the amplitude, $A$, and phase, $\varphi$, of the signal $x_{2}(t)$.

\section{COMPARISON OF INTEGRATION, CORRELATION AND DFT APPROACHES}

\subsection{Simulation results}

To compare the effectiveness of integration method, correlation method and DFT method, a simulation of these three methods was performed in NI LabView ${ }^{[14]}$. The results of this simulation are given in Tables 1-3 and in Figure 6. It was found that, for an ideal signal, the correlation method exhibits the best performance for measuring amplitude and phase of a vector signal (see Tables 1-3).

The measurement error decreases with the increase of sampling frequency. However, when noise was present, this situation reversed dramatically. In the presence of noise, the correlation method gave the worst results. Figure 6 shows the variation of amplitude and phase errors with sampling frequency for a noisy signal of the form $x(\mathrm{t})=A \sin (2 \pi f t+\varphi)+N_{1}(t)$, where $A=10 \mathrm{~V}, \varphi=30^{\circ}, f=742.857 \mathrm{kHz}$, and $N_{1}(t)$ is uniform white noise with amplitude $A_{\mathrm{N} 1}=1 \mathrm{~V}$. The correlation method was used with $y(t)=B \sin (2 \pi f t+\varphi)+N_{2}(t)$, where, $B=5 \mathrm{~V}, \varphi=60^{\circ}, f=742.857 \mathrm{kHz}$, and $N_{2}(t)$ is uniform white noise with amplitude $\mathrm{A}_{\mathrm{N} 2}=1 \mathrm{~V}$, noise $\mathrm{N}_{1}(\mathrm{t})$ and $\mathrm{N}_{2}(\mathrm{t})$ are not related, $x(\mathrm{t})$ and $y(\mathrm{t})$ are signals of two cycles. This figure shows that the DFT method provides the best prediction of amplitude and phase. Its error decreases rapidly when the sampling frequency is increased. The integration method also shows a decreasing error with increasing sampling frequency. The trend is comparable with that of the DFT method, but not as good. The correlation method is very sensitive to noise, and its error remains high even when the sampling frequency is increased to high values. This behavior of the correlation method is due to the error incurred by the calculation of signal amplitudes with Equation (14) and (15) using the autocorrelation function. The noise concentrate at the point $\tau=0$ of the correlation function, i.e., in the autocorrelation function. This error may be reduced when using the second peak value of autocorrelation function of sinusoidal signal instead of using the first peak ${ }^{[15]}$.

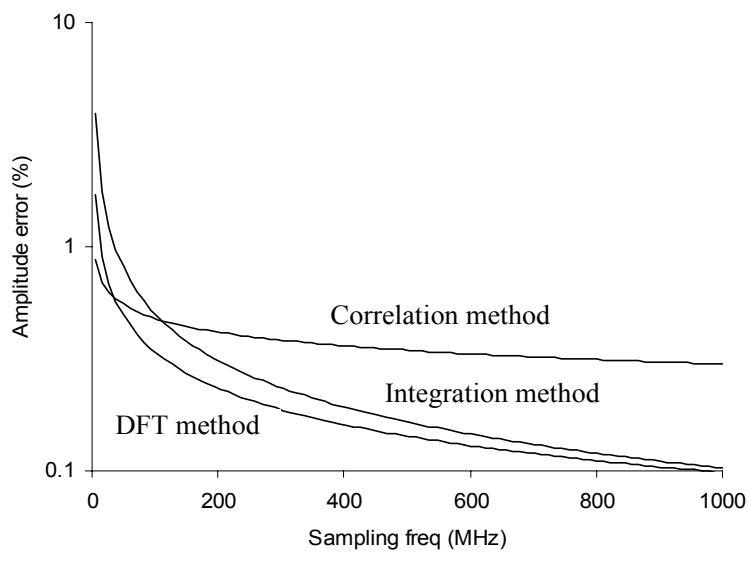

(a)

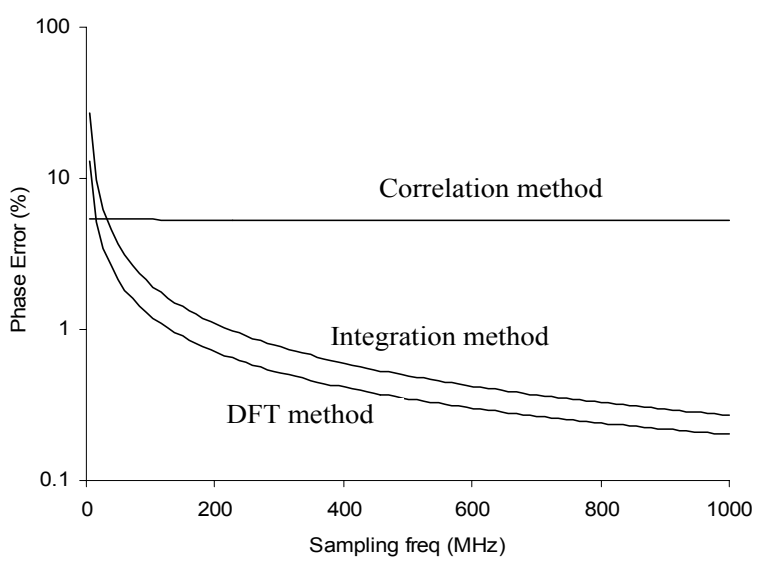

(b) 
Table 1 Integration Method, $x(t)=A \sin \left(2 \pi f t+\varphi_{1}\right)$ where, $A=10 \mathrm{~V}, \varphi_{1}=30^{\circ}, f=742.857 \mathrm{kHz}$

\begin{tabular}{|c|c|c|c|c|c|}
\hline \multicolumn{2}{|c|}{ Input } & \multicolumn{4}{c|}{ Output } \\
\hline $\begin{array}{c}\text { Sampling } \\
\text { freq fs }\end{array}$ & $\begin{array}{c}\text { BufferSize } \\
N\end{array}$ & \multicolumn{2}{c|}{ Amplitude $A$} & \multicolumn{2}{c|}{ Initial Phase $\varphi$} \\
\cline { 3 - 6 } & Value & Error (\%) & Value & Error (\%) \\
\hline $50 \mathrm{GH}$ & 2692 & 9.995 & 0.042 & 29.957 & 0.141 \\
\hline $250 \mathrm{MHz}$ & 1346 & 9.992 & 0.078 & 29.921 & 0.263 \\
\hline $100 \mathrm{MHz}$ & 673 & 9.992 & 0.079 & 29.920 & 0.264 \\
\hline $50 \mathrm{MHz}$ & 269 & 9.977 & 0.22 & 29.774 & 0.752 \\
\hline $25 \mathrm{MHz}$ & 135 & 9.927 & 0.72 & 29.126 & 2.911 \\
\hline $10 \mathrm{MHz}$ & 67 & 9.913 & 0.86 & 29.065 & 3.115 \\
\hline $5 \mathrm{MHZ}$ & 27 & 9.896 & 1.03 & 29.144 & 2.853 \\
\hline
\end{tabular}

Table 2 Correlation Method, $x(t)=A \sin \left(2 \pi f t+\varphi_{1}\right), y(t)=A \sin \left(2 \pi f t+\varphi_{2}\right)$ where, $A=10 \mathrm{~V}, \varphi_{1}=60^{\circ}, \varphi_{2}=30^{\circ}, f=742.857 \mathrm{kHz}$

\begin{tabular}{|c|c|c|c|c|c|}
\hline \multicolumn{2}{|c|}{ Input } & \multicolumn{4}{c|}{ Output } \\
\hline $\begin{array}{c}\text { Sampling } \\
\text { freq fs }\end{array}$ & $\begin{array}{c}\text { BufferSize } \\
\text { N }\end{array}$ & \multicolumn{2}{c|}{ Amplitude $A$} & \multicolumn{2}{c|}{ Initial Phase $\varphi$} \\
\cline { 3 - 6 } & Value & Error (\%) & Value & Error (\%) \\
\hline $500 \mathrm{MHz}$ & 2692 & 9.99971 & 0.00283 & 29.99998 & 0 \\
\hline $250 \mathrm{MHz}$ & 1346 & 9.99971 & 0.00280 & 29.99996 & 0.0001 \\
\hline $100 \mathrm{MHz}$ & 673 & 9.99972 & 0.00276 & 29.99993 & 0.0002 \\
\hline $50 \mathrm{MHz}$ & 269 & 9.99807 & 0.01929 & 29.99857 & 0.0047 \\
\hline $25 \mathrm{MHz}$ & 135 & 10.00640 & 0.06407 & 30.004591 & 0.0153 \\
\hline $10 \mathrm{MHz}$ & 67 & 9.99363 & 0.06369 & 29.967795 & 0.1073 \\
\hline $5 \mathrm{MHz}$ & 27 & 10.00136 & 0.01367 & 30.035276 & 0.1175 \\
\hline
\end{tabular}

Table 3 DFT Method, $x(t)=A \sin \left(2 \pi f t+\varphi_{1}\right)$ where, $A=10 \mathrm{~V}, \varphi_{1}=30^{\circ}, f=742.857 \mathrm{KHz}$

\begin{tabular}{|c|c|c|c|c|c|}
\hline \multicolumn{2}{|c|}{ Input } & \multicolumn{4}{|c|}{ Output } \\
\hline \multirow{2}{*}{$\begin{array}{l}\text { Sampling } \\
\text { freq., } f_{s}\end{array}$} & \multirow{2}{*}{$\begin{array}{c}\text { Buffer size } \\
N\end{array}$} & \multicolumn{2}{|c|}{ Amplitude $A$} & \multicolumn{2}{|c|}{ Initial Phase $\varphi$} \\
\hline & & Value & Error (\%) & Value & Error (\%) \\
\hline $1 \mathrm{GHz}$ & 2692 & 10.000288 & 0.00288384 & 29.955973 & 0.146757 \\
\hline $500 \mathrm{MHz}$ & 1346 & 10.000291 & 0.00290690 & 29.955996 & 0.14668 \\
\hline $250 \mathrm{MHz}$ & 673 & 10.000295 & 0.00295288 & 29.956042 & 0.146526 \\
\hline $100 \mathrm{MHz}$ & 269 & 10.002307 & 0.0230709 & 29.671965 & 1.09345 \\
\hline $50 \mathrm{MHz}$ & 135 & 9.991629 & 0.0837052 & 31.089352 & 3.63117 \\
\hline $25 \mathrm{MHz}$ & 67 & 10.014642 & 0.146417 & 28.283935 & 5.72022 \\
\hline $10 \mathrm{MHz}$ & 27 & 9.987396 & 0.12604 & 31.039468 & 3.46489 \\
\hline $5 \mathrm{MHz}$ & 13 & 10.115542 & 1.15542 & 18.9636015 & 36.788 \\
\hline
\end{tabular}

The integration method can directly find the real part and imaginary part of a vector signal by signal integration. Due to the present of numerical integration, this method has precision limitation even in simulation. The precision is affected dramatically by the integration step, which is controlled by the sampling frequency of the data acquisition (DAQ) device. A high sampling frequency DAQ device is need for this method. The integration method can also be viewed as a subset of correlation method: the signal is correlated with two synchronous reference signals, one of which is in phase, while the other is shifted $90^{\circ}$ out of phase. In addition, the noise level can also be significantly reduced by the selection of the appropriate integration time. Hence, this method can identify very small signals in the presence of very high levels of harmonics and noise $\mathrm{e}^{[4,16]}$.

The correlation method can precisely find the phase difference of two ideal sinusoidal signals with same frequency. However, when noise is presented, this method has big error for amplitude and phase detection. Moreover, this method can only measure the relative phase between two sinusoidal waveforms. For measuring the impedance of capacitive 
device (such as PWAS), additional work has to be done to determine the sign of the calculated impedance at the frequency points where rapid phase shifts occur.

The Fourier transform method is a powerful tool for analyzing and measuring stationary signals. It transforms samples of the data from time domain to frequency domain and has the advantages of selecting proper frequency and suppressing noise and disturbance harmonics.

Note that in all the above three methods the length of sampled signal was selected to be an integer number of cycles. For the DFT method, this avoids frequency leakage. If the FFT (fast Fourier transform) algorithm is to be used, another requirement appears: the time record should contain $2^{n}$ samples, where $n$ is an integer. To achieve this, the sampling frequency of the DAQ device should always be a power of 2 times the signal frequency. This shows that the FFT algorithm is not always desirable for all the impedance measurement applications.

\subsection{Experimental results}

\subsubsection{Experimental setup}

The practical implementation of the compact $\mathrm{E} / \mathrm{M}$ impedance analyzer concept was performed using standard low-cost multipurpose laboratory equipments: a function generator, a PCI DAQ card ${ }^{[17]}$, a PCI GPIB card, a calibrated resistor $(100 \Omega)$ and a PC with LabView software package (Figure 7). The function generator is controlled by the PC via the GPIB card. It outputs sinusoidal excitation within a defined frequency range $\left(f_{\text {start }}\right.$ to $f_{\text {end }}$, say, $100 \mathrm{kHz}$ to $\left.1 \mathrm{MHz}\right)$. The actual excitation and response of the device under test (DUT) are recorded by DAQ card and then analyzed by LabView. The resulting output is the DUT impedance over the given frequency range.

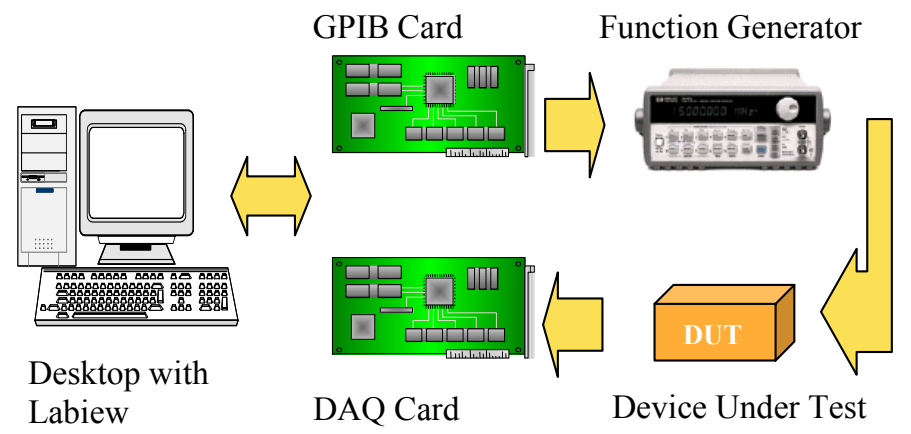

Figure 7 Desktop implementation of impedance analyzer

\subsubsection{Comparison of experimental results}

These experiments allowed us to compare the three methods (integration method, correlation method, and DFT method) against the laboratory-scale HP4194 impedance analyzer, which the dedicated instrument for impedance measurements. In our experiments we consider an active DUT consisting of a piezoelectric wafer active sensor (PWAS). PWAS is an active device due to the piezoelectric coupling between the electrical and mechanical energies that takes place in the piezoelectric materials. PWAS present electromechanical resonances and antiresonances. At antiresonance, the real part of the impedance goes through a peak, while the imaginary part of the impedance goes through zero. When mechanically free, the 7-mm diameter PWAS used in this experiment has its first in-plane antiresonance at around 350 kHz. Figure 8 and Figure 9 show the superposed results obtained with the above methods and the HP4194A laboratory impedance analyzer. DFT method and integration method are nearly indistinguishable in results. Both of these two methods work as well as HP4194 impedance analyzer. The cross correlation method shows significant error at low frequencies. This is due to the fact that the SNR is small at low frequency. Hence, the correlation method introduces considerable error in the measurement of the real part of PWAS impedance at low frequency(Figure 8). This error diminishes as the frequency increases. For the imaginary part of the PWAS impedance, the correlation method shows significant error at the antiresonance frequency. The positive/negative peaks of the imaginary part of the PWAS impedance measured by the correlation method are much larger than the those measured with the other methods (Figure 9). The cause of this discrepancy requires further attention in future research. 


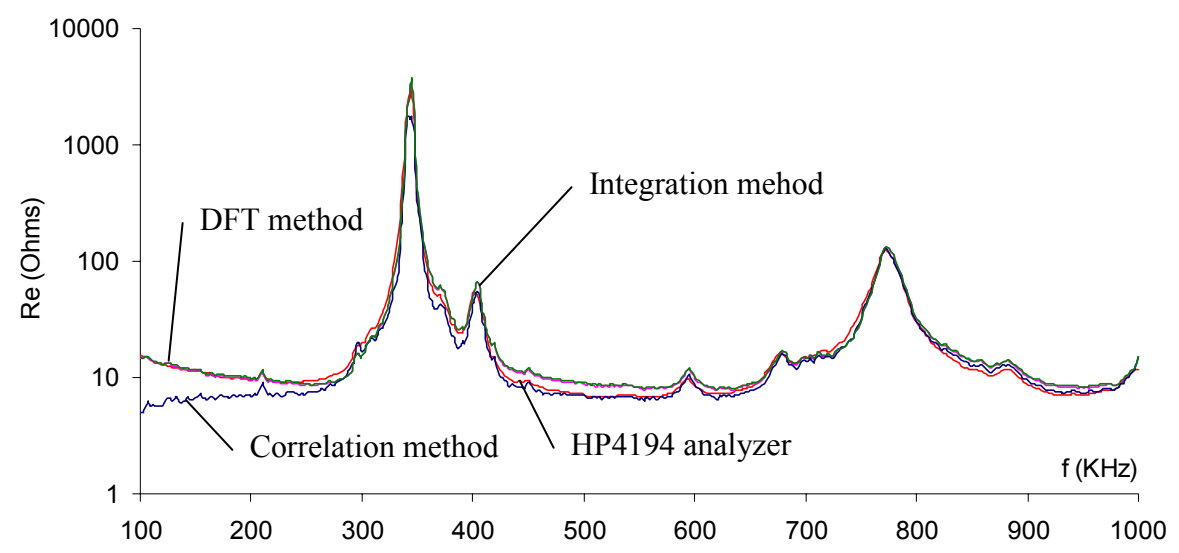

Figure 8 Comparison of measurement of real part of impedance of PWAS with different methods

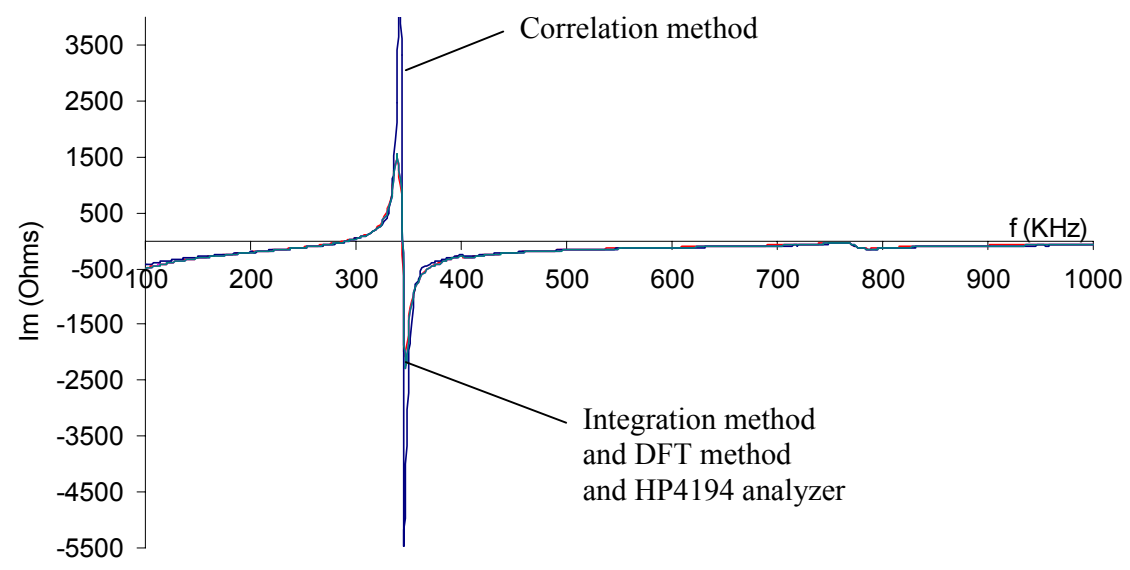

Figure 9 Comparison of measurement of imaginary part of impedance of PWAS with different methods

\section{CONCLUSION AND FURTHER WORK}

A novel concept of E/M impedance analyzer has been presented in this paper. Several data analysis methods (Integration method, Correlation method, DFT method) for impedance measurement were studied in this paper. Simulation studies on signals with added noise showed that the DFT method gives the best performance; its error decreased rapidly with increasing the sampling frequency. The integration method also performed well, but with less accuracy than the DFT method. The correlation method had the worse performance when noise was present. In the presence of noise, the error of the correlation method could not be reduced in spite the fact that the sampling frequency was increased. The experimental results for measuring the impedance of a piezoelectric wafer active sensor (PWAS) with DFT method and the signal integration method are comparable to the results obtained with HP4194 impedance analyzer. Since this digital impedance analyzer will be mainly used for structural health monitoring in conjunction with PWAS, the results are quite encouraging.

The impedance analyzer was constructed as a virtual instrument. The hardware system architecture of compact E/M impedance analyzer consists of several blocks: (a) reference signal generation-function generator; (b) voltage and current measurements-DAQ card; and (c) digital signal acquisition and processing-PC with Labview. To further reduce the size and improve the mobility of the system, the PC can be replaced by a laptop. Accordingly, a PCMCIA DAQ 
card should be used. If function generator is controlled via RS232 cable, The GPIB card can be deleted from the system. By using a digital signal processor (DSP) chip for signal analyzing instead of a personal computer and generating the sinusoidal excitation with integrated direct digital synthesis (DDS) (such as the AD9833 chip $^{[18]}$, which can provide digitally created sine wave up to $12.5 \mathrm{MHz}$ ) and sampling voltage and current signals with $\mathrm{A} / \mathrm{D}$ converter and some

peripheral circuits (e.g., tele-transmitter module ${ }^{[19,20]}$ ), a small field-portable impedance analyzer for SHM could be developed.

\section{ACKNOWLEDGMENTS}

Support from the Air Force Research Lab through UTC Contract \#03-S470-033-C1 of F33615-01-D-5801 is thankfully acknowledged.

\section{REFERENCES}

1. Giurgiutiu, V.; Xu, B. (2003) "CEMIA - Compact Electromechanical Impedance Analyzer for Structural Health Monitoring", USC-IPMO, Disclosure ID No. 00407 of 09/28/2003

2. Agilent Technologies (2003), Impedance Measurement Handbook (2nd Edition), Agilent Technologies Co. Ltd., Dec 2003.

3. QuadTeck (2003), Application Note, "The Application of Digital Signal Processing (DSP) Techniques to Precision LCR Measurement”, QuadTech, July 2003

4. http://www.solartronanalytical.com/technicalpages/techimped.html

5. http://www.gamry.com/Products/Gamry_EIS_Measurement/Gamry_EIS_Measurement.htm

6. Pardo de Vera, C.; Guemes, J. A. (1997) "Embedded Self-Sensing Piezoelectric for Damage Detection," Proceedings of the International Workshop on Structural Health Monitoring, September 18-20, 1997, Stanford, CA 445-455

7. Peairs, D.M.; Park, G; Inman, D.J. (2002) "Low Cost Impedance Monitoring Using Smart Materials", Proceeding of the First European Workshop on Structural Health Monitoring, Ecole Normale Superieure, Cachan (Paris), France, July 10-12, 2002

8. Inman, D.J. (2003) "Smart Materials in Damage Detection and Prognosis", Key Engineering Materials Vol. 245-246 (2003) pp3-16

9. Park, G.; Sohn, H.; Farrar, C. R.; Inman, D. J. (2003), "Overview of Piezoelectric Impedance-Based Health Monitoring and Path Forward", The Shock and Vibration Digest, November, 2003

10. Peairs, D.M.; Park, G; Inman, D.J. (2004) "Improving Accessibility of the impedance-Based Structural Health Monitoring Method", Journal of Intelligent Materials Systems and Structures, Vol. 15, 129-139, Feb. 2004

11. Agilent application note 1405-2, Understanding Dynamic Signal Analysis, Agilent Technologies Co. Ltd.

12. Darowicki, K.; et al (2003), "Continuous-frequency Method of Measurement of Electrode Impedance", Instrumentation Science \& Technology, Vol 31, No. 1, pp 53-62, 2003

13. Evans, N.J.; Sayers, B. (1994), "Recent advances in electrochemical impedance measurement", Engineering in Medicine and Biology Society, 1994. Engineering Advances: New Opportunities for Biomedical Engineers. Proceedings of the 16th Annual International Conference of the IEEE, Pages:A72 Vol.1 Nov. 1994

14. Johnson, G.W. (1998), LABVIEW power programming, McGraw-Hill

15. Liu, JH (2003), Labview programming for virtual instrument, Publishing House of Electronics Industry, Beijing, China, Jan. 2003

16. Macdonald, J. R.(1987), Impedance spectroscopy emphasizing solid materials and systems, Johm Wiley \& Sons, Inc., 1987

17. http://www.gageapplied.com/

18. http://www.analog.com/UploadedFiles/Data_Sheets/37150134474160AD9833_a.pdf

19. Lynch, J.P.; Law, K.H. (200), "The Development of a wireless Modular Health Monitoring System for Civil Structures", Proceedings of the MCEER Mitigation of Earthquake Disaster by Advanced Technologies (MEDAT-2) Workshop, Las Vegas, NV, USA, November 30-31, 2000

20. Tanner, N.; Farrar, C. R.; Sohn, H. (2002), "Structural health monitoring using wireless sensing systems with embedded processing", Proceedings of SPIE Vol. \#4704, 18-19 March 2002 